\title{
Aerosol Droplet Surface Measurement Methods
}

\author{
Derrick M. MotT, Mao FukuYama, and Akihide Hibara ${ }^{\dagger}$ \\ Institute of Multidisciplinary Research for Advanced Material (IMRAM), Tohoku University, \\ IMRAM West Building 1, Room S211, 2-1-1 Katahira, Aoba, Sendai 980-8577, Japan
}

\begin{abstract}
Aerosol droplets play a critical role in the development of weather patterns, yet are notoriously difficult to analyze because of their small size, transient nature and potentially complex composition. As a result, there has been a surge in recent years in the development of analysis techniques aimed at the study of aerosol droplets, namely of their surface tension properties, which are thought to play a great role in aerosol/cloud growth and subsequently having an impact on the resulting weather patterns. To capture the state of the field at this key time, we have collected and described some of the most relevant and influential studies, with a focus on those that have had the most impact. This review will present and describe the most used analytical techniques for studying the surface tension of micrometer-sized aqueous droplets, with a focus on historical trends and how the current techniques are posed to revolutionize the field.
\end{abstract}

Keywords Aerosol, sessile, surface tension, atmospheric analysis, quasi-elastic laser scattering

(Received May 28, 2020; Accepted July 15, 2020; Advance Publication Released Online by J-STAGE July 24, 2020)

1 Introduction

2 Theory and Current State of the Field

$2 \cdot 1$ Köhler theory

2.2 Experimental limitations and challenges:

Traditional oversimplification of results

$2 \cdot 3$ Objectives in clarifying the current state of the field

3 Current Approaches and Challenges in Measuring

Surface Tension of Atmospheric Aerosols

3.1 Pendant drop measurement
61

62

3.2 Atomic force microscopy

3.3 Calculation based approaches

3.4 Visualization of surface oscillation

3.5 Optical tweezers and induced deformation measurement

3.6 Spontaneous capillary wave resonance and light scattering measurement

4 Summary and Outlook

5 Acknowledgements

6 References

\section{Introduction}

Weather prediction is a critically important field that plays a role in agriculture, aviation/travel, energy production, climate change prediction, etc. Even though this field has been studied since ancient times, the primary mechanisms of cloud formation and evolution still have many mysteries. ${ }^{1-3}$ Clouds are made of

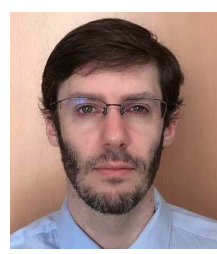

Derrick MoTT received his Ph.D. in 2008 from Department of Materials Science at the State University of New York at Binghamton. He worked as a JSPS postdoctoral research fellow at the Japan Advanced Institute of Science and Technology (2009 - 2010), before becoming an Assistant Professor there $(2010$ - 2018). He then Professor. She works at Institute of Multidisciplinary became a Project Assistant Professor at Tohoku Research for Advanced Materials, Tohoku University at the Institute of Multidisciplinary University as an Assistant Professor (2017 -). Her Research for Advanced Materials (2018 -). His research interests are micro- and nanofluidic research interests focus on nanoscale size effects, systems for chemical and biochemical applications, environmental monitoring, sensing technologies liquid interface science. and materials science.

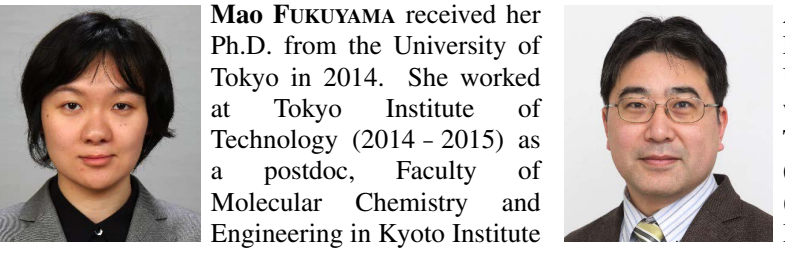

Akihide HiBara received his Ph.D. in 2003 from the University of Tokyo. He worked at the University of Tokyo as Research Associate (1999 - 2003), Lecturer (2003 - 2007), and Associate Professor $(2007-2013)$. He worked at Tokyo Institute of Technology as Associate Professor (2013 - 2016). Now, he works as Professor at Institute of Multidisciplinary Research for Advanced Materials, Tohoku University (2016-). He serves as an Editor of Sensors and Actuators B: Chemical and an Associate Editor of Chemistry Letters. His research interest is microfluidic chemical and bioanalysis, and laser spectroscopy.

† To whom correspondence should be addressed.

E-mail: hibara@tohoku.ac.jp 
aerosols, nanometer- to micrometer-sized droplets composed of liquid and solid debris held aloft in the air. The mechanism of cloud formation is generally understood and is the result of moist air and particulate matter rising from the earth's surface to lower pressure altitudes, which causes expansion of the air and subsequent condensation of the moisture to form an aerosol. The conventional and well-known droplet vapor pressure model that governs this will be introduced in Sect. 2.

The formation of aerosol from a weather perspective is challenging to predict and model however, because the processes that govern the aerosol formation are poorly understood. ${ }^{1-3}$ For example, the aerosol can be a complex mixture composed of water, salts, organic molecules and inorganic materials, or any combination of these, which will affect not only the rate of aerosol formation, but other properties such as albedo of the resulting cloud. ${ }^{4-6}$

It has recently been revealed that one of the most important pieces of information is the effect of surface tension of the aerosol droplet on the resulting droplet growth. ${ }^{7}$ While traditional studies assumed that the surface tension of most atmospheric aerosols was equal to that of water, new results have shown that the presence of various surfactants or other materials in the atmosphere may be contributing to surface tension depression of the droplets, ${ }^{1,8}$ which impact the cloud formation rate and have a large influence on the resulting localized weather. Understanding and predicting this effect is one important aspect that is required to improve weather modelling and prediction. The first step, however, is accurately measuring the surface tension of liquid droplets in the nano- to micrometer-size range, which is a significant experimental challenge.

Over recent years, there have been many attempts to accurately measure the surface tension of both bulk liquid and their small size scale counterparts to elucidate the roll surface tension plays in aerosol droplet formation. The most traditional approach is the pendant drop method, which was used by Noziere et al. to determine the surface tension of liquids containing re-dissolved surfactants captured from the atmosphere. ${ }^{9}$ The study showed new insight into the role that surfactants might play in modifying surface tension, but failed to incorporate the effect of small droplet sizes. Atomic force microscopy (AFM) was successfully employed by Morris et al. to determine the surface tension of sub-micron sized sessile droplets in a humid environment, ${ }^{10}$ which was an early success in measuring surface tension for such small droplets, but the results relied heavily on the substrate, so could not be directly applied to spherical suspended droplets. Dutcher et al. used a calculational approach to estimate surface tension for small sized aerosol droplets, ${ }^{6}$ a method that is unencumbered by physical limitations in handling small aerosol droplets, but is also restricted by the accuracy of the model used in the calculation. Ishiwata et al. successfully demonstrated the use of a modulated jet stream to create aerosol droplets that were analyzed with strobe photography to visualize surface oscillations and subsequently calculated the resulting surface tension. ${ }^{11}$ The technique was straightforward, but suffered from the inability to monitor transient surface tension properties. A powerful breakthrough was realized when optical tweezers began to be employed by Bzdek et al. for capturing suspended aerosol droplets and measuring their surface tension through oscillatory effects, ${ }^{12,13}$ which revealed unique size effects on the surface tension of aerosols. However, the technique was limited in the ability to only analyze one droplet at a time under static environmental conditions. Finally, one of the most recent attempts to measure surface tension incorporates the use of an optical tweezer to capture aerosol droplets, while the surface tension is monitored through the quasi elastic laser scattering (QELS) technique. Hibara et al. successfully demonstrated the capture and surface tension measurement for aerosol droplets using a laser-based optical tweezer system, while surface tension under different surfactant conditions was monitored using QELS. ${ }^{14,15}$

The current state of the field is expected to pave the way to more advanced studies using the above described systems to monitor the real-time effect of surface tension in aerosol droplets under different environmental conditions, leading to an expanded understanding of how atmospheric conditions influence aerosol/ cloud formation and the resulting impact on climate, weather patterns and weather prediction. The present review will provide an overview of the background of this critical field, followed by a discussion of the primary techniques that have been used to investigate surface tension in relation to aerosol analysis, leading up to the most advanced techniques used today and the direction the field is expected to take in the future.

\section{Theory and Current State of the Field}

\subsection{Köhler theory}

Aerosol droplet growth is described by the Köhler equation, which was introduced in 1936 by Hilding Köhler and describes the process in which water vapor condenses and forms liquid drops. ${ }^{16}$ The theory is based upon equilibrium thermodynamics and combines the Kelvin effect which describes the change in saturation vapor pressure due to a curved surface and Raoult's Law, which relates the saturation vapor pressure to the solute. The theory has been instrumental in describing atmospheric processes and phenomena, particularly cloud formation and evolution, which is important for accurate weather prediction. ${ }^{1,2}$ Equation (1) shows the Köhler equation. Note the important role that surface tension $(\sigma)$ plays in the equation and as a result, the rate of aerosol droplet growth.

$$
\ln \left(\frac{p_{\mathrm{w}} D_{\mathrm{p}}}{p^{0}}\right)=\frac{4 M_{\mathrm{w}} \sigma_{\mathrm{w}}}{R T \rho_{\mathrm{w}} D_{\mathrm{p}}}-\frac{6 n_{\mathrm{s}} M_{\mathrm{w}}}{\pi \rho_{\mathrm{w}} D_{\mathrm{p}}^{3}}
$$

Where $p_{\mathrm{w}}$ is droplet water vapor pressure, $p^{0}$ is the corresponding saturation vapor pressure over a flat surface, $\sigma_{\mathrm{w}}$ is the droplet surface tension, $\rho_{\mathrm{w}}$ is the density of pure water, $n_{\mathrm{s}}$ is moles of solute, $M_{\mathrm{w}}$ is the molecular weight of water and $D_{\mathrm{p}}$ is the cloud drop diameter.

\subsection{Experimental limitations and challenges: Traditional oversimplification of results}

As can be observed in the Köhler equation, the surface tension $\left(\sigma_{\mathrm{w}}\right)$ term plays an important role in the gas-liquid equilibrium of cloud droplets, yet the surface tension value for pure water has long been used, even if the liquid contains various solutes. ${ }^{2,3}$ This approach has traditionally assumed that the distribution of all dissolved substances is uniform throughout the droplet, which may be an oversimplification as described by several recent studies. ${ }^{1,2,8}$ In addition, the measurement of surface tension effects for dilute dissolved substances in water has been performed at the bulk scale, which may not be an accurate representation of the small-scale size effects that can occur at the micrometer scale for liquid droplets. The forming aerosol water droplets begin at the nano to submicrometer scale, where size effects can strongly influence the surface tension. In addition, technological constraints have limited the direct observation and measurement of aerosol droplet growth in an in-situ environment. ${ }^{8}$ The small size of developing aerosol 

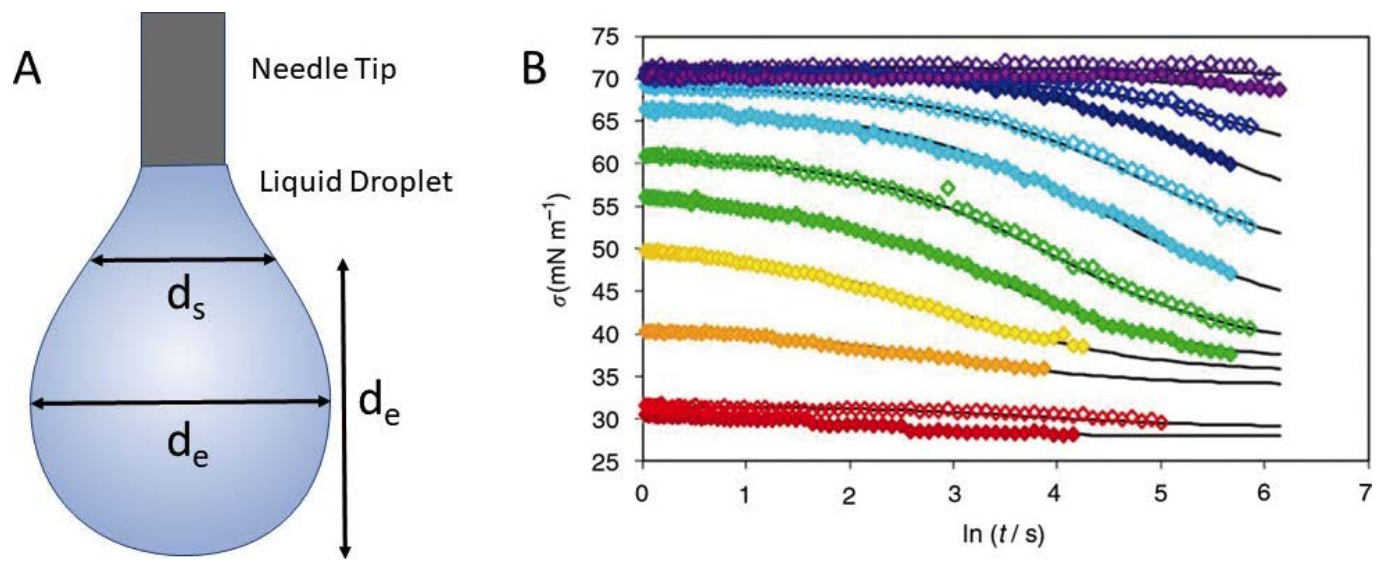

Fig. 1 Scheme illustrating the general pendant drop technique for surface tension measurement (A), surface tension curves for an increasingly diluted atmospheric sample showing how meso-equilibration time varies based on surfactant concentration (B). Figure 1B reproduced with permission from (B. Nozière, C. Baduel, and J. L. Jaffrezo, Nat. Commun., 2014, 5, 3335) under Creative Commons License. Published by Springer Nature.

droplets makes it incredibly challenging to directly measure the surface tension, as well as the composition of individual micrometer-sized droplets. ${ }^{17-19}$ Current techniques rely on indirect measurements, such as determining the composition of an accumulated aerosol droplet sample.

\subsection{Objectives in clarifying the current state of the field}

The field of aerosol science and cloud formation dynamics is evolving quickly because of an enhanced understanding of the role that natural organic surfactants and inorganic salts play in affecting the surface tension of aerosol droplets. In addition, recent advances in the area of technical limitations for measuring micrometer-sized droplet composition and surface tension are expected to reveal the missing piece of the Köhler theory puzzle for predicting droplet growth in an atmospheric environment. In response, this review seeks to provide a synopsis of the current techniques used to measure the surface tension of aerosol droplets and give a concise appraisal of the new techniques in the field and their expected impact on the study of aerosol droplet formation, as well as the role that organic surfactants or inorganic salts play in modifying surface tension.

\section{Current Approaches and Challenges in Measuring Surface Tension of Atmospheric Aerosols}

As the field of aerosol droplet analysis has advanced, it has become increasingly necessary to develop a technique to assess the surface tension of aerosol-sized droplets (diameter less than $1 \mu \mathrm{m})$. It has been predicted that the presence of surfactants or solutes in developing aerosol droplets leads to surface tension depression, which in turn affects the rate of droplet growth. ${ }^{3}$ The phenomenon though is poorly understood, and the direct role that surfactants play in the surface tension depression can only be indirectly inferred at this point. To elucidate the situation, direct measurement and continuous monitoring of the surface tension of single aerosol droplets is required, which is challenging because of the small size of the droplets involved. The following sections describe the techniques and corresponding studies that have utilized aerosol droplet surface tension measurement, discussing their strengths and weaknesses, and then introduces new techniques that have been developed to elucidate the role of surface tension by focusing on characterization of individual aerosol droplets.

\section{3·1 Pendant drop measurement}

Perhaps the most commonly used and well understood technique for determining the surface tension of a liquid is the pendant drop method, where suspension of a liquid droplet at the end of a tube and the force of surface tension is proportional to the length of the boundary between the tube and droplet. The result is a straightforward equation relating the mass of the droplet to the tube diameter and surface tension (i.e. $m g=\pi d \gamma$, where $m$ is mass, $g$ is acceleration of gravity, $d$ is the tube diameter and $\gamma$ is surface tension). In practical terms, however, when the pendant drop method is used to determine an unknown surface tension, the formula $\gamma=\Delta \rho g d_{\mathrm{e}}(1 / H)$, where $\Delta \rho$ is a density difference and $H$ is a correction factor derived from reference values, is used. Figure 1A shows a general scheme illustrating the pendant drop method.

One of the influential studies on the effect of surfactants on surface tension depression was performed by Nozière et al. ${ }^{9}$ In this work, the authors collected aerosol samples in Grenoble, France using a high-volume sampler, and then the surfactant was extracted through a separation process. The isolated surfactant was re-dissolved in pure water, which was then used in determining the surface tension of submillimeter droplets using the pendant drop measurement technique. The study revealed new information in the role that surfactants play on aerosol surface tension, as shown in Fig. 1B, yet suffered from limitations. Namely, the pendant drop method cannot be used for very small droplet sizes less than about $1 \mathrm{~mm}$ because the droplet shape becomes distorted by the tube used for creating the droplet, or in other words, the droplet shape becomes dominated by surface tension effects as opposed to gravity, making the measurement technique inaccurate for small droplet sizes. As a result, the determined surface tensions may not reflect the true value of those for aerosol-sized droplets, which exist in the submicron to micron size range.

\subsection{Atomic force microscopy}

Atomic force microscopy (AFM) has been used for many years to image the submicron morphology of surfaces and for 


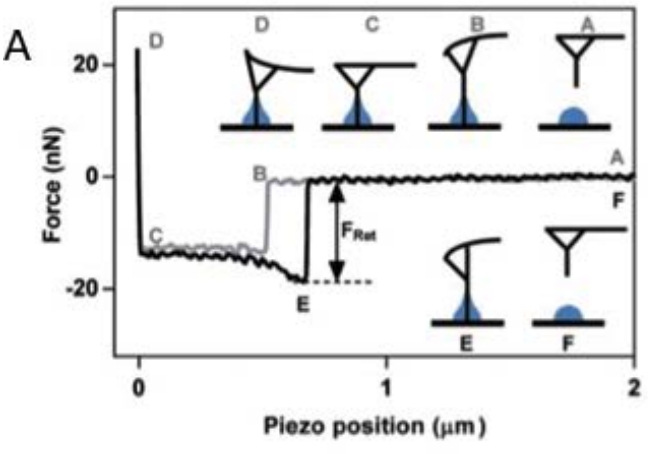

B

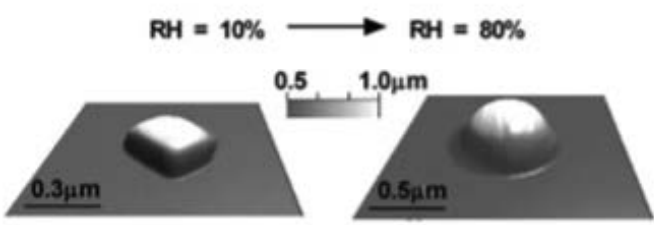

C

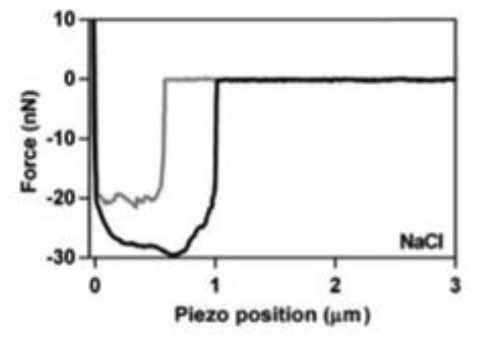

$\mathrm{D}$

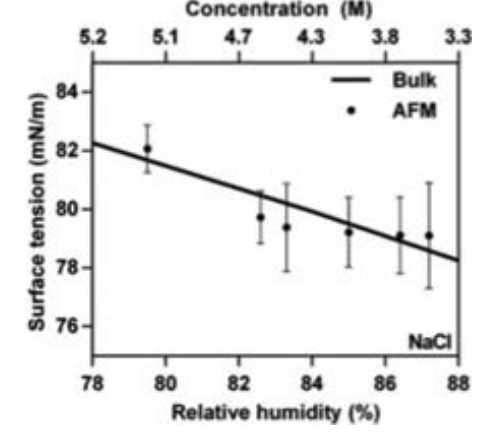

Fig. 2 Scheme of force-based AFM tip interaction with droplet to determine surface tension (A), and experimental measurement results of a sodium chloride containing droplet (B), force profile (C) and resulting humidity dependent surface tension (D). Figure 2 adapted with permission from (H. S. Morris, V. H. Grassian, and A. V. Tivanski, Chem. Sci., 2015, 6, 3242). Published by the Royal Society of Chemistry.

probing the surface properties in terms of chemical nature or hydrophobicity/hydrophilicity. In the work by Morris et al. ${ }^{10}$ AFM is used in conjunction with finely fabricated silver-gallium tips to probe the surface tension of micron-sized droplets containing salt and organic molecules. The surface tension measurement is performed not by imaging, but by measuring the AFM tip force profile, while positioning the needle close to a droplet on a substrate, and then retracting the needle as shown in Fig. 2. The retention force (force required to free the needle from the meniscus during retraction) was measured and then was used to calculate surface tension with the following formula:

$$
F_{\text {Ret }}=2 \pi \sigma r
$$

where $F_{\text {Ret }}$ is the retention force, $r$ is tip radius and $\sigma$ is surface tension. In their experiments, solid materials such as salt, glutaric acid and malonic acid were deposited onto a substrate. Then, the humidity was raised until deliquescence occurred (condensation of moisture on the solid materials and their dissolution). The surface tension was measured for each type of droplet several times at different humidity levels, but for all samples high humidity had to be maintained so that all the material remained dissolved. Typically, humidity levels greater than $75 \%$ were used. Sodium chloride containing droplets showed a surface tension value of $79-82 \mathrm{mN} / \mathrm{m}$, slightly elevated from that for pure water. In contrast, glutaric acid droplets showed a surface tension value that ranged from about $40-50 \mathrm{mN} / \mathrm{m}$ dependent on humidity, while malonic acid also showed a value of about $40-50 \mathrm{mN} / \mathrm{m}$, both greatly depressed from the measured value of sodium chloride containing droplets.

\subsection{Calculation based approaches}

In the realm of calculation-based approaches to the determination of surface tension for aerosol droplets, thermodynamic modelling has been employed, utilizing bulk data of simple solutions to estimate the surface tension of more complex solutions or liquid configurations. ${ }^{6}$ For example, in the work performed by Dutcher et al., the surface tension was modelled for both liquid-vapor and liquid-liquid interfaces through thermodynamic modelling and by analyzing interfacial tension of biphasic microscale flows. Such an approach brought new insight and information to interpreting the surface tension effects occurring within more complex aerosol structures, such as those with phase separated organic and aqueous components, or those containing solid material within the aerosol as shown in Fig. 3A. In one example of modelled results, the surface tension of solutions of sodium chloride, glutaric acid and their mixture was compared to those that were experimentally measured (Fig. 3B). The modelled data showed a good fit to the experimental values of surface tension, and was extrapolated to ranges where experimental measurements could not be performed such as for very high concentrations. ${ }^{6}$ Calculationbased approaches are advantageous because of the flexibility they offer in studying aerosols with complex structures and predicting the expected properties, including for surface tension. However, the accuracy of the modelled results is dependent on the accuracy of the model used, which makes a direct comparison between the model and environmentally measured results a challenge. Additional drawbacks to the modelling technique for estimating surface tension of aerosols include the lack of incorporation of small size (tens of microns) effects in the modelling of the surface tension.

\subsection{Visualization of surface oscillation}

One of the earliest used tools for studying the surface tension of droplets in the micrometer size range is the direct visualization and analysis of the droplets surface oscillation. This has been achieved for aqueous droplets in air by ejecting a stream of liquid from a nozzle, which is formed into a string of droplets by a piezoelectric actuator. ${ }^{11,20}$ The droplets are typically analyzed by strobe lighting and image capture by a high speed camera or CCD. The images are used to visualize the distorted 

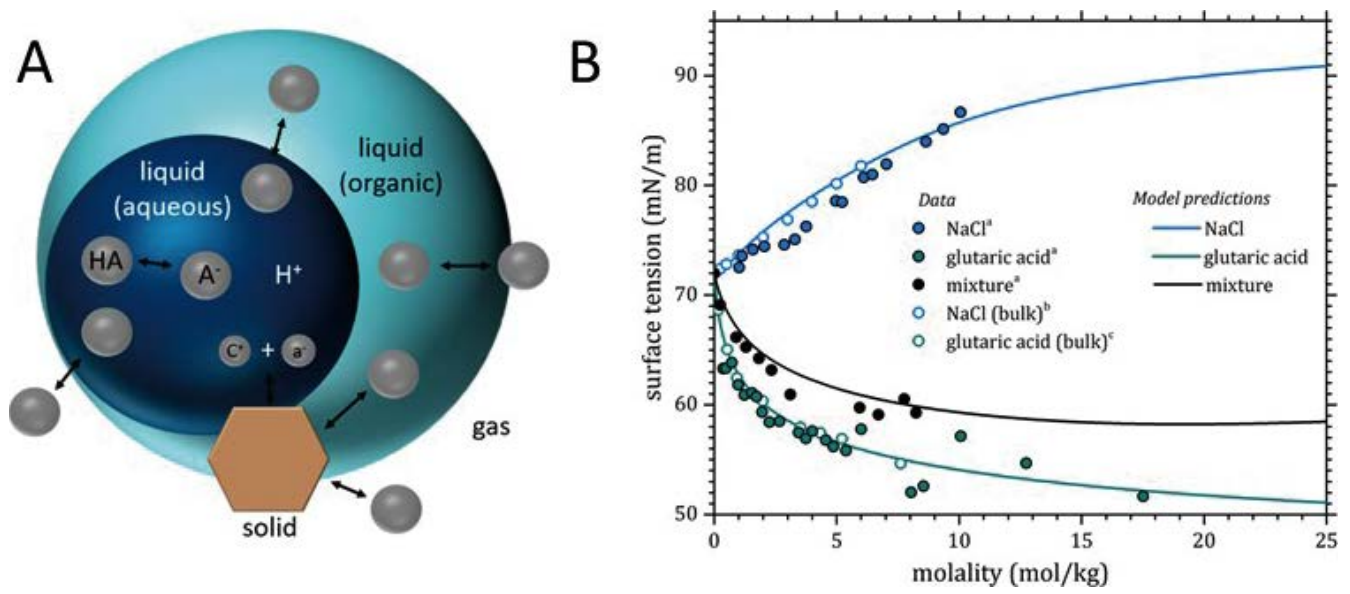

Fig. 3 Schematic illustration of the structure and processes occurring within a multi-structured aerosol droplet (A), and the surface tension of solutions of $\mathrm{NaCl}$, glutaric acid and a mixture of the two, where empty circles represent bulk measurements, closed circles are measurements of micrometer sized droplets using optical tweezers, and the solid lines are the modelled values (B). Adapted with permission from (H. C. Boyer and C. S. Dutcher, J. Phys. Chem. A, 2017, 121, 4733). Copyright (2017) American Chemical Society.

A

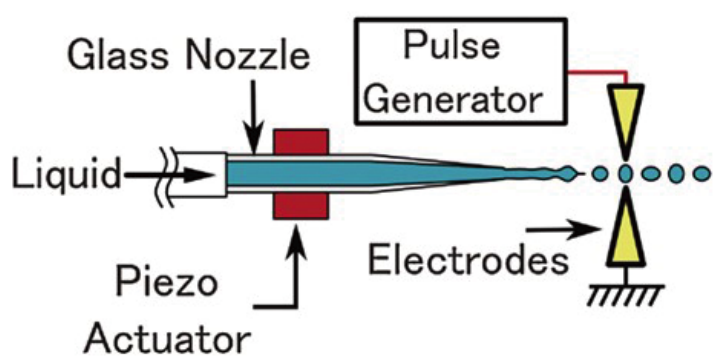

B

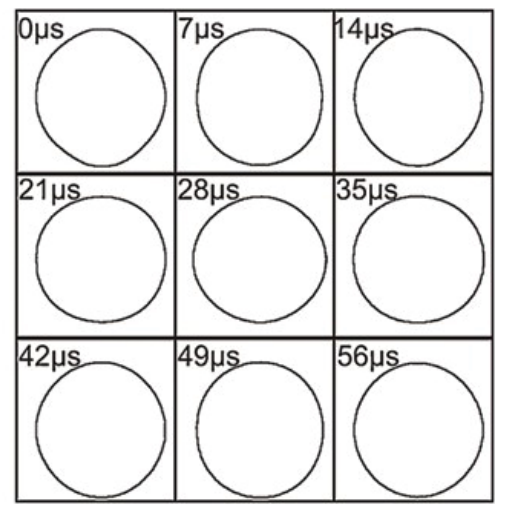

Fig. 4 Generation of oscillating micro-droplets using a piezo-actuator with droplet deformation achieved by an applied electric field (A). An oscillating droplet is imaged using strobe photography and the droplet shape is outlined (B), which is then used to calculate the surface tension. Copyright (2014) The Japan Society of Applied Physics.

droplet shape during the oscillation, which is then used to numerically calculate the resulting surface tension. Ishiwata et al. successfully used this approach to measure the surface tension of droplets composed of water and sodium decylsulfate solution. ${ }^{11}$ In this work, they improved the analytical technique by incorporating an electric field across the formed droplets to control the excitation of oscillations, shown in Fig. 4A, which in turn lead to highly reproducible droplet shapes in the analysis of the oscillation derived surface tension. Figure $4 \mathrm{~B}$ shows an outline of the droplet over time and illustrates how the shape of the droplet changes due to surface oscillations.

This general technique has also been extended to other liquiddroplet systems, such as flowing aqueous droplets through immiscible liquid flows in a PDMS device to determine interfacial surface tension. ${ }^{21,22}$ Hudson et al. analyzed the interfacial surface tension of both air and aqueous droplets suspended in a wide range of fluids, including ethylene glycol, glycerol and various silicone fluids, ${ }^{21}$ while Metcalf et al. utilized a similar device-driven approach to analyze the surface tension of aqueous droplets containing surfactants, including ammonium sulfate and methylglyoxal. ${ }^{22}$ These approaches all revealed new information in the behavior of surface tension for micrometer size droplets, but suffer from drawbacks such as little flexibility in changing droplet size, or limitations in monitoring surface tension change when exposed to different reactants.

\subsection{Optical tweezers and induced deformation measurement}

Optical tweezers have generated interest in many fields as a sensitive technique that allows the immobilization and manipulation of materials in the micrometer and smaller size domain. ${ }^{23-29}$ The technique relies on a focused laser where a strong electric field exists at the laser focal point, allowing dielectric materials to be immobilized or trapped. Because of the non-invasive nature of optical trapping, it is an attractive technique for studying the surface tension properties of aerosol droplets. Recent studies by Bzdek et al. demonstrate the trapping of droplets with a micrometer size radius and determination of surface tension through the observation of 

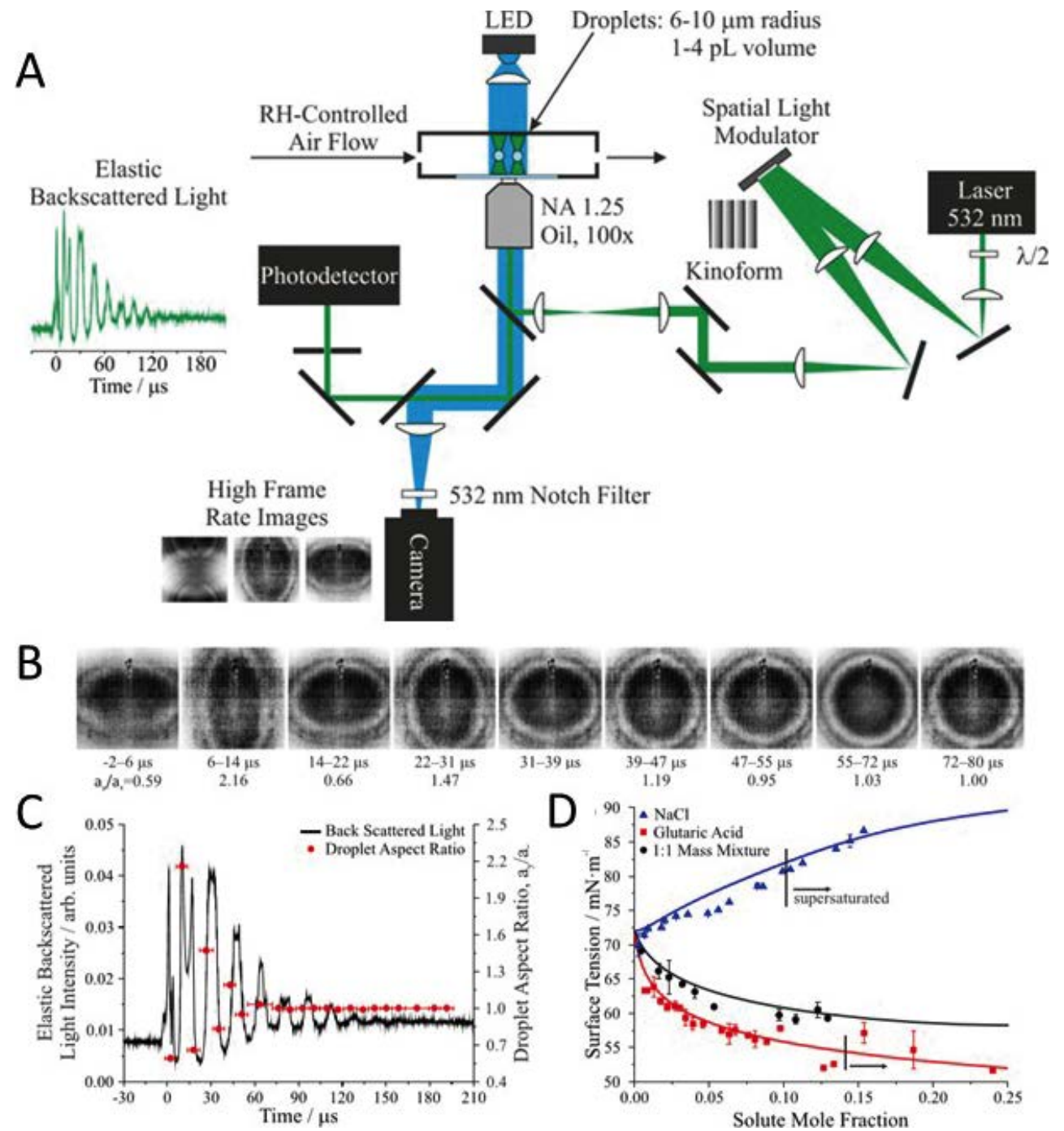

Fig. 5 Illustration of the optical tweezer trapping technique and droplet coalescence to study surface tension properties. The technique relies on multiple laser light optical traps whose contents are then allowed to coalesce (A). The resulting oscillation of the merged droplet is observed with a high-speed camera (B). Surface tension information is extracted through modelling of the droplet dynamics (C, D). Figure 4A reprinted from (B. R. Bzdek, L. Collard, J. E. Sprittles, A. J. Hudson, and J. P. Reid, J. Chem. Phys., 2016, 145, 054502), with the permission of AIP Publishing. Figures 4B - 4D reprinted from (B. R. Bzdek and J. P. Reid, J. Chem. Phys., 2017, 147, 220901), with the permission of AIP Publishing.

oscillation after the coalescence of multiple droplets. The oscillation is detected by reflection intensity of the laser beam. ${ }^{12,13,30,31}$ The oscillation is then modelled to extract the surface tension and viscosity values. Figure $5 \mathrm{~A}$ shows an overview of the optical trapping technique used in Bzdek's study where a focused laser is used to immobilize two droplets with controllable size and composition. The droplets are then merged to cause oscillation, as shown in Fig. 5B. The oscillation is monitored with a photodetector in Fig. 5C, which is used to determine the surface tension of the merged droplets containing sodium chloride, glutaric acid, or a mixture of the two (Fig. 5D). The technique successfully demonstrates differences in surface tension between droplets containing different surfactants. While the technique is highly sensitive and reveals key information on the surface properties of micrometer-sized droplets, it is not applicable to single droplet analysis or monitoring of the properties of a single droplet, leaving several challenges to be solved.

\subsection{Spontaneous capillary wave resonance and light scattering measurement}

The previously discussed techniques all set out to elucidate the role of surface tension in atmospherically relevant aerosol droplets, yet all face some form of limitation, either in terms of the scale of the measurement (the droplets analyzed are much larger than the micrometer size range of atmospheric aerosols), or the technique physically interacts with the droplet, affecting the accuracy and relevance of the measurement. As a result, a technique is greatly needed that can analyze the surface tension (and influence of environmental molecules) of micrometer-sized droplets in a non-invasive way. Recently, this has become possible due to advances in the use of optical tweezers ${ }^{12,13,30,31}$ to immobilize micrometer-sized aerosols (non-invasive) and the use of capillary-sphere wave resonance to probe the surface tension characteristics. ${ }^{14,15}$

The recent study "Spherical Spontaneous Capillary-Wave Resonance on Optically Trapped Aerosol Droplet" by Endo 

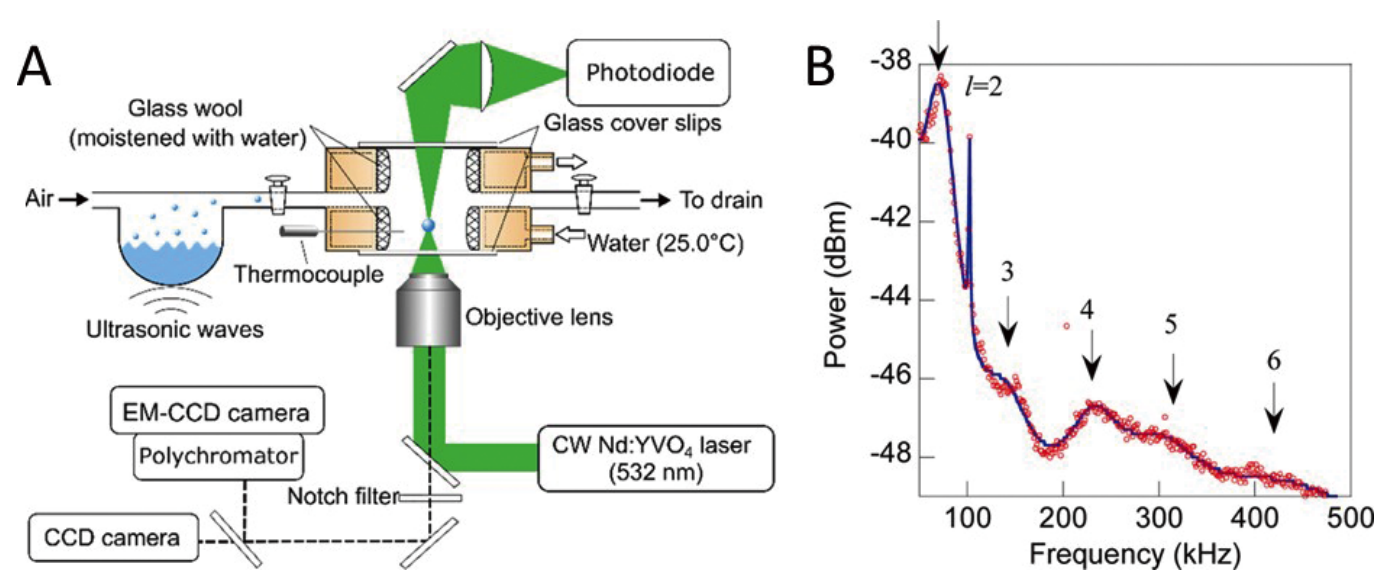

Fig. 6 Schematic illustration of the experimental setup for aerosol droplet trapping using optical tweezers and surface resonance measurement using QELS (A), and an example QELS spectrum taken using this system for a 12.4 micrometer radius aerosol droplet, revealing several resonance modes in the spectrum, which can be used to determine the surface tension. Reprinted with permission from ( $\mathrm{T}$. Endo, K. Ishikawa, M. Fukuyama, M. Uraoka, S. Ishizaka, and A. Hibara, J. Phys. Chem. C, 2018, 122, 20684). Copyright (2018) American Chemical Society.

et al. details the technique and how it may be employed in the study of the surface tension of atmospheric aerosol droplets. ${ }^{14,15}$ The technique relies on two phenomena to achieve aerosol droplet capture and surface tension analysis, the first is the use of a focused laser that acts as an optical tweezer, and the second is the use of quasi-elastic laser scattering (QELS). ${ }^{32-40}$ Spontaneous spherical resonance occurs as a result of thermal fluctuations at the surface of the liquid droplet, which can be observed in the $\mathrm{Hz}$ range of frequencies in a QELS spectrum. Figure 6 shows the experimental setup used to immobilize aerosol droplets using an optical tweezer as well as the use of QELS to analyze the spherical resonance modes at the droplet surface. These resonance modes were used to calculate the surface tension of the droplet through Lambs equations. ${ }^{15,41}$ The result is a system that has the ability to isolate and analyze a single aerosol droplet, isolating surface tension effects. The system was successfully used to measure the surface tension of droplets as small as $4.7 \mu \mathrm{m}$ radius, containing the organic surfactants sodium dodecylsulfate and ammonium sulfate, revealing a depressed surface tension value.

\section{Summary and Outlook}

The field of atmospheric surface science is rapidly evolving, making new techniques and new thinking highly necessary to solve the challenges being introduced in the field. Historically speaking, the study of atmospheric science has been conducted "outside" of the laboratory, focusing on the direct observation of weather-related phenomena, or collecting atmospheric/ environmental samples for in-lab characterization. It is becoming increasingly important to incorporate complementary techniques to these, such as calculational approaches, or creating model systems in the laboratory for direct analysis to promote advancements in the field of atmospheric science. Such approaches can lead to breakthroughs by directing the environmental studies or identifying fundamental phenomena to isolate and characterize. The critical ability to study aerosol droplets with controllable size and composition is integral to finally solving the relationship between aerosol droplet surface tension, and subsequent growth of the droplet in a real-world atmospheric environment. In this regard, the quickly developing field of optical trapping of aerosols and their surface tension characterization using QELS are positioned to become invaluable for revealing new insight and information on atmospheric phenomena and cloud formation. Initial studies have proven the viability of the technique, and future work is likely to improve the analysis in terms of trapping smaller droplets and/or monitoring droplet surface tension before, during and after exposure to various organic surfactants. These approaches and advances will fill the gap in the understanding of the role that surface tension plays in the field of atmospheric science, while also opening new avenues in the study of this intriguing and valuable scientific area.

\section{Acknowledgements}

This work was supported by the Japan Society for the Promotion of Science (JSPS) KAKENHI (grant no 18H03913) and by the Research Program of the "Dynamic Alliance for Open Innovation Bridging Human, Environment and Materials" in the "Network Joint Research Center for Materials and Devices".

\section{References}

1. J. Ovadnevaite, A. Zuend, A. Laaksonen, K. J. Sanchez, G. Roberts, D. Ceburnis, S. Decesari, M. Rinaldi, N. Hodas, M. C. Facchini, J. H. Seinfeld, and C. O'Dowd, Nature, 2017, 546, 637.

2. A. Kroflič, S. Frka, M. Simmel, H. Wex, and I. Grgić, Environ. Sci. Technol., 2018, 52, 9179.

3. C. R. Ruehl, J. F. Davies, and K. R. Wilson, Science, 2016, 351, 1447.

4. U. Pöschl and M. Shiraiwa, Chem. Rev., 2015, 115, 4440.

5. M. Shiraiwa, K. Ueda, A. Pozzer, G. Lammel, C. J. Kampf, A. Fushimi, S. Enami, A. M. Arangio, J. Fröhlich-Nowoisky, Y. Fujitani, A. Furuyama, P. S. J. Lakey, J. Lelieveld, K. Lucas, Y. Morino, U. Pöschl, S. Takahama, A. Takami, H. Tong, B. Weber, A. Yoshino, and K. Sato, Environ. Sci. Technol., 2017, 51, 13545. 
6. H. C. Boyer and C. S. Dutcher, J. Phys. Chem. A, 2017, $121,4733$.

7. B. Noziere, Science, 2016, 351, 1396.

8. A. Schwier, D. Mitroo, and V. F. McNeill, Atmos. Environ. 2012, 54, 490.

9. B. Nozière, C. Baduel, and J. L. Jaffrezo, Nat. Commun., 2014, $5,3335$.

10. H. S. Morris, V. H. Grassian, and A. V. Tivanski, Chem. Sci., 2015, 6, 3242.

11. T. Ishiwata and K. Sakai, Appl. Phys. Express, 2014, 7, 077301.

12. B. R. Bzdek, R. M. Power, S. H. Simpson, J. P. Reid, and C. P. Royall, Chem. Sci., 2016, 7, 274.

13. B. R. Bzdek, L. Collard, J. E. Sprittles, A. J. Hudson, and J. P. Reid, J. Chem. Phys., 2016, 145, 054502.

14. T. Endo, K. Ishikawa, M. Fukuyama, and A. Hibara, in 21st International Conference on Miniaturized Systems for Chemistry and Life Sciences, MicroTAS 2017, 2017, $599-600$.

15. T. Endo, K. Ishikawa, M. Fukuyama, M. Uraoka, S. Ishizaka, and A. Hibara, J. Phys. Chem. C, 2018, 122, 20684.

16. H. Köhler, Trans. Faraday Soc., 1936, 32, 1152.

17. S. Enami, Y. Sakamoto, and A. J. Colussi, Proc. Natl. Acad. Sci. U. S. A., 2014, 111, 623.

18. S. Ishizuka, T. Hama, and S. Enami, J. Phys. Chem. C, 2019, 123, 21662.

19. S. Ishizuka, A. Matsugi, T. Hama, and S. Enami, J. Phys. Chem. Lett., 2020, 11, 67.

20. B. Stückrad, W. J. Hiller, and T. A. Kowalewski, Exp. Fluids, 1993, 15, 332.

21. S. D. Hudson, J. T. Cabral, W. J. Goodrum, K. L. Beers, and E. J. Amis, Appl. Phys. Lett., 2005, 87, 081905.

22. A. R. Metcalf, H. C. Boyer, and C. S. Dutcher, Environ. Sci. Technol., 2016, 50, 1251 .

23. A. Ashkin and J. M. Dziedzic, Appl. Phys. Lett., 1971, 19 , 283.

24. A. Ashkin and J. M. Dziedzic, Appl. Phys. Lett., 1974, 24 586.

25. A. Ashkin and J. M. Dziedzic, Science, 1975, 187, 1073.

26. A. Ashkin, Science, 1980, 210, 1081.

27. S. Ishizaka, Y. Suzuki, and N. Kitamura, Phys. Chem. Chem. Phys., 2010, 12, 9852.

28. S. Ishizaka, J. Ma, T. Fujiwara, K. Yamauchi, and N. Kitamura, Anal. Sci., 2016, 32, 425.

29. M. Uraoka, K. Maegawa, and S. Ishizaka, Anal. Chem., 2017, 89, 12866.

30. B. R. Bzdek and J. P. Reid, J. Chem. Phys., 2017, 147, 220901.

31. B. R. Bzdek, J. P. Reid, J. Malila, and N. L. Prisle, Proc. Natl. Acad. Sci. U. S. A., 2020, 117, 8335.

32. S. Takahashi, A. Harata, T. Kitamori, and T. Sawada, Anal. Sci., 1994, 10, 305.

33. Y. Ikezoe, S. Ishizaki, H. Yui, M. Fujinami, and T. Sawada, Anal. Sci., 2004, 20, 1509.

34. Y. Uchiyama, H. Yui, and T. Sawada, Anal. Sci., 2004, 20, 1537.

35. H. Yui, Y. Ikezoe, and T. Sawada, Anal. Sci., 2004, 20, 1501.

36. T. Nomoto, T. Toyota, and M. Fujinami, Anal. Sci., 2014, 30, 707 .

37. M. Ohno, T. Toyota, T. Nomoto, and M. Fujinami, Anal. Sci., 2015, 31, 979.

38. A. Hibara, M. Nonaka, M. Tokeshi, and T. Kitamori, J. Am. Chem. Soc., 2003, 125, 14954.

39. M. Chung, C. Pigot, S. Volz, and A. Hibara, Anal. Chem., 2017, 89, 8092.

40. C. Pigot and A. Hibara, Anal. Chem., 2012, 84, 2557.

41. H. Lamb, "Hydrodynamics", 1932, 6th ed., Cambridge University Press, Cambridge. 